\title{
Embolus-carried vascular endothelial cell growth factor 165 improves angiogenesis in thromboangiitis obliterans
}

\author{
Q. Liu ${ }^{1 *}$, H.C. Zhou ${ }^{2 *}$, X.L. Yang ${ }^{1}$, Y.P. Zhao ${ }^{1}$, H.P. Luo ${ }^{1}$, C.W. He ${ }^{1}$ and \\ X.B. Wan ${ }^{1}$ \\ ${ }^{1}$ The First Affiliated Hospital of Nanchang University, Nanchang, China \\ ${ }^{2}$ Yingtan People's Hospital, Yintan, China \\ *These authors contributed equally to this study. \\ Corresponding author: X.L. Yang \\ E-mail: lqdocchina@126.com / lq99001@163.com
}

Genet. Mol. Res. 13 (1): 1744-1752 (2014)

Received December 22, 2012

Accepted August 6, 2013

Published March 17, 2014

DOI http://dx.doi.org/10.4238/2014.March.17.2

\begin{abstract}
We investigated neovasculization effects of emboluscarried human vascular endothelial cell growth factor $165\left(\mathrm{VEGF}_{165}\right)$ encoded adenovirus (Ad) vector in the hindlimbs of rats with thromboangiitis obliterans (TAO). Rats were equally divided into blank control (I), TAO model (II), embolus (III), Ad-VEGF ${ }_{165}$ intravascular treatment (IV), Ad-VEGF ${ }_{165}$ intramuscular treatment (V), and emboluscarried Ad-VEGF ${ }_{165}$ (VI) groups. After interventional treatment, the neovasculization effect of the test gene was observed using immunohistochemistry. At 1 week after administration, compared with group II, groups V and VI had significantly increased microvessel densities, but no significant difference was observed between groups $\mathrm{V}$ and VI. At 2 weeks, groups V and VI exhibited significantly increased microvessel densities. At 1 week after administration, compared with group II, both groups V and VI showed a significant difference in the ratio between the $\alpha$-smooth muscle actin count and the muscle fiber count, whereas no significant difference was observed between them. At 2 weeks, groups V and VI also exhibited significant differences in these
\end{abstract}


ratios compared with the other groups. We conclude that Ad-VEGF ${ }_{165}$ promotes neovasculization in ischemic limbs. Embolus-carried AdVEGF $_{165}$ had the most pronounced effect.

Key words: Femoral artery; Vascular endothelial cell growth factor; Embolus; Neovascularization; Gene therapy

\section{INTRODUCTION}

Thromboangiitis obliterans (TAO), first reported by Von Winiwater in 1879, is a common peripheral arterial chronic occlusive disease, as well as the most common vascular disease responsible for amputations in the prime of life. TAO is characterized by high disability and recurrence rates, thus seriously influencing the patient's life and work. Once TAO is confirmed, timely treatment should be given. To date, interventional therapy, Chinese traditional treatment based on syndrome differentiation, vasodilating agents, and surgical therapy have been adopted for TAO. For surgical therapy, forms of lumbar sympathectomy, thrombus intimectomy, bypass operation, great omentum grafting, and staged arteriovenous shunts (i.e., venous arterialization) may be performed. However, when TAO develops into a serious condition in which blood flow reconstruction and angioplasty fail, therapeutic neovascularization is likely to provide a new idea. Therapeutic neovascularization is a technique that promotes neovascularization in ischemic regions by applying vascular growth factor or its encoding gene, aimed at increasing hemoperfusion. Vascular endothelial growth factor (VEGF) is one of the most powerful and specific growth factors associated with vascular growth. VEGF selectively promotes vascular endothelial cell (VEC) division, where it plays a vital regulatory role in angiogenesis and angiopoiesis and strongly promotes VEC proliferation, stimulating neovascularization. Furthermore, it has the virtues of easy use, extensive application, and good safety, thereby occupying an irreplaceable treatment position. Although empirical and clinical studies of the application of VEGF in the treatment of chronic lower limb ischemia have been reported, how to increase the expression of the VEGF gene in ischemic tissues remains an unsolved issue.

In this study, the angiogenesis-promoting effect of the $\mathrm{VEGF}_{165}$ gene in the ischemic rat hindlimb was explored by transporting $\mathrm{VEGF}_{165}$-carrying emboli through the vessels.

\section{MATERIAL AND METHODS}

\section{Animals}

A total of 72 adult SD rats weighing $300 \pm 50 \mathrm{~g}$ were supplied by the experimental animal center of Medical School of Nanchang University. This study was carried out in strict accordance with the recommendations in the Guide for the Care and Use of Laboratory Animals of the National Institutes of Health. The animal use protocol was reviewed and approved by the Institutional Animal Care and Use Committee of Nanchang University.

\section{Methods}

TAO rat models were established using the modified method of Zhou et al. (2002). A 
blood sample of $0.5 \mathrm{~mL}$ was obtained from each model and then mixed with $500 \mu \mathrm{LVEGF}_{165}$ plasmid-containing solution. After coagulation, the thrombus formed was placed on a 35-mesh screen and pressed repeatedly to sift it through. A suspension of $\mathrm{VEGF}_{165}$-carrying emboli was prepared, with each embolus having a diameter of about $0.5 \mathrm{~mm}$. Constructed adenovirus (Ad)-VEGF ${ }_{165}$ was used to infect HEK293 cells. After virus amplification, the cells were subpackaged and stored at $-80^{\circ} \mathrm{C}$. Titers were determined using limiting dilution assays for $100 \mathrm{x}$ $10^{8} \mu \mathrm{L}$ plasmids per dosage.

The animals were equally divided into groups I, II, III, IV, V, and VI. Group I was the normal control group and group II was the TAO control group. Neither of them received interventional therapy. Group V was injected with $0.5 \mathrm{mLAd}-\mathrm{VEGF}_{165}$ plasmid suspension at five sites of the muscles near the femoral artery stump $(0.1 \mathrm{~mL}$ at each). Groups III, IV, and VI received injections of $0.5 \mathrm{~mL}$ non-VEGF ${ }_{165}$-carrying embolus, Ad-VEGF ${ }_{165}$ plasmid, and VEGF ${ }_{165}$-carrying embolus suspensions into the femoral artery, respectively. At 7 and 14 days after operation, six rats from each group were taken for muscle tissue specimens. VECs and smooth muscle cells were respectively detected with CD34 and $\alpha$-smooth muscle actin (SMA) antibodies. Microvessels were counted in 200X fields for microvessel density (MVD). Five fields were set for each specimen and means were calculated. $\alpha$-SMA-positive capillaries and skeletal muscle fibers were counted in five 400X fields and their mean ratio was obtained (Tanigawa et al., 1997).

\section{Statistical analysis}

All data are reported as means \pm SD and analyzed by the SPSS18.0 software. Oneway ANOVA was performed, and least significant difference (LSD) tests were used to compare means between groups. $\mathrm{P}<0.05$ was considered to be statistically significant.

\section{RESULTS}

\section{CD34}

CD34 expression was observed using immunohistochemistry. At 1 week after therapy, CD34 was expressed in all specimens: capillary endothelial cells were stained buffy to brown with surrounding buffy positive granules. Compared with group II, both groups V and VI showed a significant difference $(\mathrm{F}=6.06, \mathrm{P}<0.05$ and $\mathrm{F}=8.21, \mathrm{P}<0.05$, respectively), whereas no significant difference was observed between them $(\mathrm{P}>0.05)$. At 2 weeks, although CD34 was still expressed in all the groups (Figures 1 and 2), it was abundantly expressed in group VI (Figure 3). Group VI exhibited a noticeable difference compared with any other group (all $\mathrm{P}<0.05$ ). The MVDs in different groups are summarized in Table 1.

\section{$\alpha$-SMA-positive capillary counts}

At 1 week, $\alpha$-SMA-positive-stained capillaries were observed in all specimens (Figures 4-6). Compared with group II, both groups V and VI showed a significant difference in the $\alpha$-SMA-positive capillary count/skeletal muscle fiber count ratio $(\mathrm{F}=15, \mathrm{P}<0.05$ and $\mathrm{F}=$ $20.66, \mathrm{P}<0.05$, respectively), but they did not exhibit a significant difference compared with each other $(\mathrm{P}>0.05)$. At 2 weeks, the $\alpha$-SMA-positive capillary count/skeletal muscle fiber 
count ratio of group VI exhibited significant differences compared with those of groups II and $\mathrm{V}(\mathrm{F}=91.71, \mathrm{P}<0.05$ and $\mathrm{F}=6.92, \mathrm{P}<0.05$, respectively $)$. The ratios in different groups are summarized in Table 2.

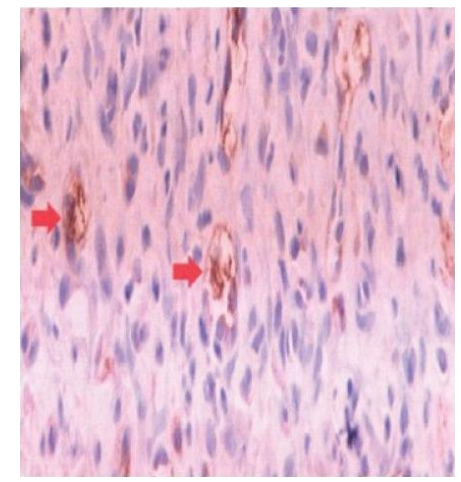

Figure 1. CD expression in the thromboangiitis obliterans control group (arrows) (200X).

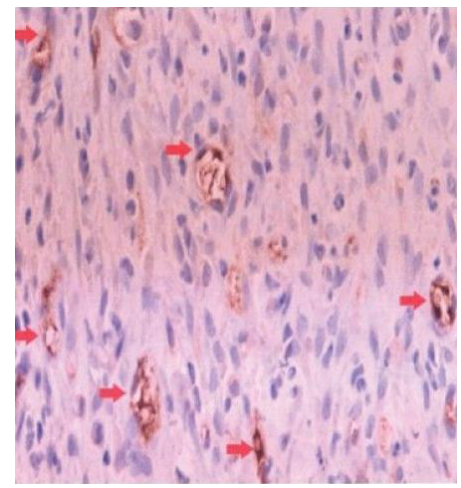

Figure 2. CD expression in the intramuscular treatment group (arrows) (200X).

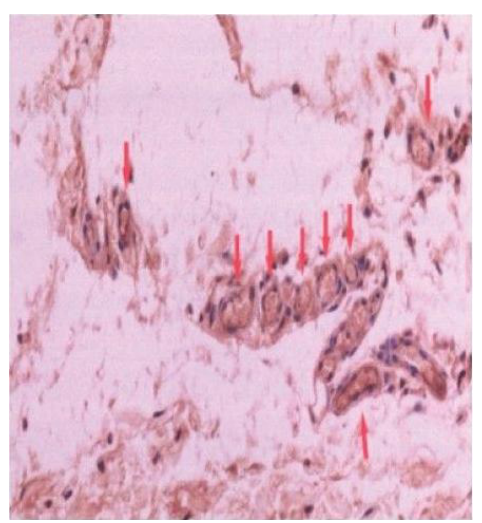

Figure 3. CD expression in the embolus-carrying group (arrows) (200X). 
Table 1. Microvessel density in different groups.

\begin{tabular}{lcr}
\hline Groups & 1 week after operation & 2 weeks after operation \\
\hline I & $28.1 \pm 3.83$ & $28.51 \pm 5.30^{*}$ \\
II & $29.59 \pm 3.39$ & $31.68 \pm 3.36$ \\
III & $30.59 \pm 3.01$ & $33.38 \pm 3.25$ \\
IV & $32.30 \pm 3.37$ & $45.69 \pm 3.11$ \\
V & $33.33 \pm 3.50$ & $49.16 \pm 4.09$ \\
VI & $34.78 \pm 4.22$ & $56.11 \pm 5.61$ \\
\hline
\end{tabular}

Data are reported as means $\pm \mathrm{SD} . * \mathrm{P}<0.05$ compared with other groups, according to an LSD test. For group explanations, see Material and Methods.

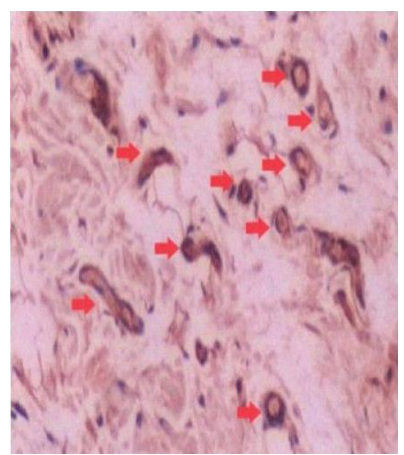

Figure 4. $\alpha$-SMA expression in the embolus-carrying group (arrows) (200X).

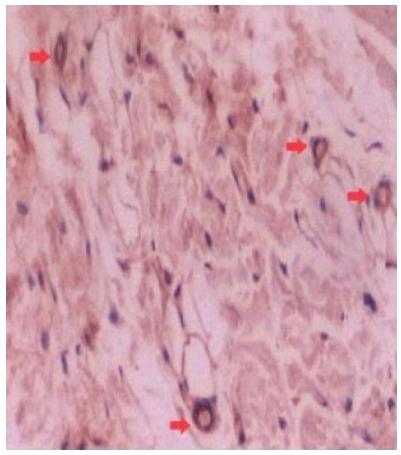

Figure 5. $\alpha$-SMA expression in the intramuscular treatment group (arrows) (200X).

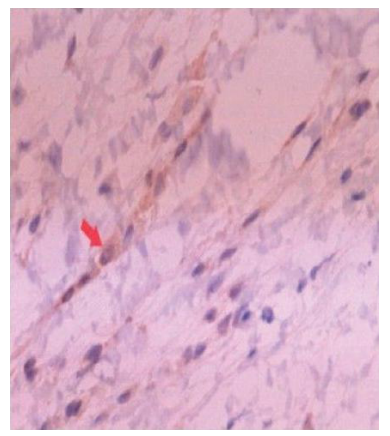

Figure 6. $\alpha$-SMA expression in the thromboangiitis obliterans control group (arrow) (200X). 
Table 2. $\alpha$-SMA-positive capillary count/skeletal muscle fiber count ratios.

\begin{tabular}{lcc}
\hline Groups & 1 week after operation & 2 weeks after operation \\
\hline I & $0.2683 \pm 0.0214$ & $0.2767 \pm 0.0403$ \\
II & $0.3035 \pm 0.0308$ & $0.2933 \pm 0.0281$ \\
III & $0.2850 \pm 0.0362$ & $0.2833 \pm 0.0163$ \\
IV & $0.3083 \pm 0.0331$ & $0.5500 \pm 0.0672$ \\
V & $0.3533 \pm 0.0493$ & $0.4533 \pm 0.0314$ \\
VI & $0.3783 \pm 0.0553$ & $0.6733 \pm 0.0931$
\end{tabular}

Data are reported as means \pm SD. For group explanations, see Material and Methods.

\section{DISCUSSION}

TAO normally involve the middle and small arteries of distal limbs, as well as their accompanying veins. It has a long disease course and can recur periodically. Once confirmed, it should be treated timely. Improving limb ischemia can prevent ischemic acral sphacelation, which leads to amputations. Therefore, for years, investigators have been searching for an effective method for promoting neovascularization in ischemic regions.

Because the major target cells or tissues of VEGF gene therapy for ischemic diseases of lower extremities are arteries and muscles, intravascular gene transfer and muscle cell gene transfer are the two means of gene transfer. Traditional methods adopt intravascular gene transfer by resorting to interventional techniques; however, these methods are difficult to perform and can lead to many complications; furthermore, they often fail in the treatment of combined-segment arterial obstruction and small artery obstructions of distal limbs (March, 1996). Comparatively, direct intramuscular injection is a new method. The first study of direct injection of reporter gene-carrying plasmid DNA into the skeletal muscles of rats was performed by Wolff et al. (1990), and the results showed that exogenous genes can be expressed in muscle cells. The gene transfer efficiency of ischemic muscles is higher than that of normal muscles; therefore, this method is a better choice for lower extremity ischemic disease (Takeshita et al., 1996). Gene transfer efficiency varies according to numerous expression factors, such as animal species, age, gender, DNA amounts, injection solvents, DNA promoters, muscular pretreatment, and so on (Wells and Goldspink, 1992; Davis et al., 1993).

VEGF is one of the most powerful and specific growth factors associated with vascular growth. It selectively promotes VEC division (Ferrara and Henzel, 1989; Connolly et al., 1989; Ferrara et al., 1992), thus playing a vital role in angiogenesis and development. It strongly promotes VEC proliferation and stimulates in vivo neovascularization by binding with the specific receptors (Flt-1 and Flt-1/KDR) on VEC surface (Takeshita et al., 1994a). Since Takeshitea et al. (1994b) proved that intra-arterial VEGF application promotes revascularization of ischemic limbs, many scholars in recent years have made attempts to enhance the neovascularization-promoting effect of VEGF by using physical techniques such as ultrasound, ion beam irradiation, and magnetic field effect. Barzelai et al. (2006) used hypointense ultrasound for ischemic hindlimb irradiation in rats and found that hemoperfusion into the ischemic tissues of moderately ischemic rats was markedly enhanced and that VEGF expression was noticeably increased. Heissig et al. (2005) used low-dose ion irradiation for ischemic rats to stimulate mastocytes to move towards the ischemic regions, which promote revascularization in those regions by secreting VEGF. Jiang et al. (2005) injected VEGF DNA naked plasmid-encapsulating magnetic nanometer particles into the artery of rats with hindlimb 
ischemia and then enriched these particles in the ischemic regions using an external magnetic field to promote neovascularization and alleviate limb ischemia. Also, gene transfer depends on other factors such as gene administration methods, viral vectors, and potential diseases (Tongers et al., 2008). Despite rather satisfactory achievements, studies on enhanced neovascularization-promoting VEGF through physical techniques are still in their infancy. In addition, interventional methods for gene transfer have a limitation in case selection. Although direct intramuscular injection is easy to perform, stability of VEGF expression remains to be increased. In contrast, target gene-carrying embolus therapy for lower extremity ischemic disease can simultaneously overcome the aforementioned limitations. From thrombosis to thrombus generation causing complete blood flow obstruction, it takes about a week. Between 8 to 14 days after thrombosis, the thrombi appear gruel-like. After 2 weeks, these thrombi adhere tightly to the vessel wall. Thrombus development is concomitant with the stimulation of the fibrinolytic system, which causes the disintegration and liquefaction of the thrombi. As a result, partial or even complete recanalization of vessels occurs. Autologous blood is extracted from animals and placed at room temperature to form thrombi for the preparation of microembolus-containing suspensions; the suspensions are injected into the animals; because the red thrombi obtained contain small amounts of fibrin, they are more vulnerable to autolysis in vivo (Sakurama et al., 1994).

Gene transfection can be accomplished by either viral or non-viral vectors. Theoretically, Ad vector induces angiogenesis to some extent (Giordano et al., 1996; Muhlhauser et al., 1996). In this study, Ad vector was transfected into the TAO rat models. The result showed that the $\mathrm{VEGF}_{165}$ gene carried by adenovirus increased the amount of neovessels. More importantly, when VEGF ${ }_{165}$ was encapsulated by thromboemboli, such an effect became particularly noticeable. The underlying mechanisms may be as follows.

1) The emboli are small enough in size. Thrombus with a diameter less than $25 \%$ of that of the femoral artery does not markedly influence the blood flow velocity and volume of the artery. Furthermore, emboli have good plasticity, which enables their morphology to change according to the shapes and sizes of vessels. This feature of emboli allows VEGF to reach the intended acral vessels that need to be treated. Within a short time, emboli occlude some small vessels and capillaries, which protects them from being washing away or dissolved by blood flow for a certain period, allowing the carried VEGF to have sufficient contact with VEC and thereby increasing gene transfer rate. Moreover, emboli benefit the even distribution of VEGF among capillaries, thereby overcoming the drawback of an uneven distribution after an intramuscular injection. After a period, emboli become smaller because of self contraction or partial dissolution. Accordingly, the VEGF gene is partially released. These smaller emboli are then driven farther into smaller vessels, extending their action.

2) Although emboli cause short-term local ischemia and anoxia to the occluded vessels, the expression of VEGF receptors increases under such a condition. Since it is an endothelial cell (EC)-specific growth factor (Connolly et al., 1989), VEGF promotes the activation, proliferation, and migration of ECs. In addition, VEGF has a chemotaxis- and migrationpromoting effect on smooth muscle cells (Li et al., 2003). This effect removes smooth muscle cells from ECs and drives them to form tubular structure under anoxia. Instead of decreasing, the expression of VEGF receptors increases.

3) Emboli may also serve as condensates for the VEGF gene. Without the solidification effect of emboli, the VEGF gene directly injected into the affected artery would soon be 
diluted by blood. Consequently, its effective concentration would decrease, and meanwhile, VEGF would not be retained in the affected limb but rather transferred to other parts of the body, leading to failure of targeted gene therapy.

Although gene therapy can improve anoxia of ischemic tissues by constructing collateral circulation, it cannot achieve normal arterial perfusion levels. Therefore, after gene therapy, pathological manifestations can still be observed with the modeled hindlimbs of TAO rats. Meanwhile, these manifestations may show persistent development treads. These would indicate the need for further treatment.

Although there are still many problems in gene therapy for ischemic disease, it is certain that these problems can be solved with advances in biotechnology and molecular biology. It is expected that the safety coefficients of gene therapy and optimal transgene protocols will be established in the near future. By then, neovascularization-associated gene therapy may bring about a new breakthrough in the prevention and cure of ischemic disease.

\section{REFERENCES}

Barzelai S, Sharabani-Yosef O, Holbova R, Castel D, et al. (2006). Low-intensity ultrasound induces angiogenesis in rat hind-limb ischemia. Ultrasound Med. Biol. 32: 139-145.

Connolly DT, Heuvelman DM, Nelson R, Olander JV, et al. (1989). Tumor vascular permeability factor stimulates endothelial cell growth and angiogenesis. J. Clin. Invest. 84: 1470-1478.

Davis HL, Whalen RG and Demeneix BA (1993). Direct gene transfer into skeletal muscle in vivo: factors affecting efficiency of transfer and stability of expression. Hum. Gene Ther. 4: 151-159.

Ferrara N and Henzel WJ (1989). Pituitary follicular cells secrete a novel heparin-binding growth factor specific for vascular endothelial cells. Biochem. Biophys. Res. Commun. 161: 851-858.

Ferrara N, Houck K, Jakeman L and Leung DW (1992). Molecular and biological properties of the vascular endothelial growth factor family of proteins. Endocr. Rev. 13: 18-32.

Giordano FJ, Ping P, McKirnan MD, Nozaki S, et al. (1996). Intracoronary gene transfer of fibroblast growth factor-5 increases blood flow and contractile function in an ischemic region of the heart. Nat. Med. 2: 534-539.

Heissig B, Rafii S, Akiyama H, Ohki Y, et al. (2005). Low-dose irradiation promotes tissue revascularization through VEGF release from mast cells and MMP-9-mediated progenitor cell mobilization. J. Exp. Med. 202: 739-750.

Jiang H, Zhang T and Sun X (2005). Vascular endothelial growth factor gene delivery by magnetic DNA nanospheres ameliorates limb ischemia in rabbits. J. Surg. Res. 126: 48-54.

Li TS, Hamano K, Nishida M, Hayashi M, et al. (2003). CD117+ stem cells play a key role in therapeutic angiogenesis induced by bone marrow cell implantation. Am. J. Physiol. Heart Circ. Physiol. 285: H931-H937.

March KL (1996). Therapy based on gene transfer methods for local gene transfer: methods for local gene delivery in the cardiovascular system. Vasc. Surg. 124: 154.

Muhlhauser J, Jones M, Yamada I, Cirielli C, et al. (1996). Safety and efficacy of in vivo gene transfer into the porcine heart with replication-deficient, recombinant adenovirus vectors. Gene Ther. 3: 145-153.

Sakurama T, Kitamura R and Kaneko M (1994). Tissue-type plasminogen activator improves neurological functions in a rat model of thromboembolic stroke. Stroke 25: 451-456.

Takeshita S, Zheng LP, Brogi E, Kearney M, et al. (1994a). Therapeutic angiogenesis. A single intraarterial bolus of vascular endothelial growth factor augments revascularization in a rabbit ischemic hind limb model. J. Clin. Invest. 93: 662-670.

Takeshita S, Pu LQ, Stein LA, Sniderman AD, et al. (1994b). Intramuscular administration of vascular endothelial growth factor induces dose-dependent collateral artery augmentation in a rabbit model of chronic limb ischemia. Circulation 90: II-228-II-234.

Takeshita S, Isshiki T and Sato T (1996). Increased expression of direct gene transfer into skeletal muscles observed after acute ischemic injury in rats. Lab. Invest. 74: 1061-1065.

Tanigawa N, Amaya H, Matsumura M, Lu C, et al. (1997). Tumor angiogenesis and mode of metastasis in patients with colorectal cancer. Cancer Res. 57: 1043-1046.

Tongers J, Roncalli JG and Losordo DW (2008). Therapeutic angiogenesis for critical limb ischemia: microvascular therapies coming of age. Circulation 118: 9-16. 
Wells DJ and Goldspink G (1992). Age and sex influence expression of plasmid DNA directly injected into mouse skeletal muscle. FEBS Lett. 306: 203-205.

Wolff JA, Malone RW, Williams P, Chong W, et al. (1990). Direct gene transfer into mouse muscle in vivo. Science 247: 1465-1468.

Zhou XH, Liu ZD, Zhu XN, Lv WY, et al. (2000). Improvement on making animal model of thromboangiitis obliterans. Liaoning J. Trad. Chin. Med. 27: 378-379. 